\title{
Open repair of juxtarenal aortic aneurysms: 10-years of experience in a single center
}

\author{
Doğan Kahraman (1), Uğur Şener (D), Tahir Olgaç®, Mehmet Moda (D) \\ Department of Cardiovascular Surgery, Gaziantep University Şahinbey Research and Application Hospital, Gaziantep, Turkey
}

\begin{abstract}
Objectives: This study aims to evaluate the long-term surgical outcomes of juxtarenal aortic aneurysms (JAAs).

Patients and methods: We retrospectively analyzed a total of 21 consecutive patients $(20$ males, 1 female; mean age $64.8 \pm 11.8$ years; range, 31 to 80 years) who underwent open repair of JAAs using a suprarenal clamp between May 2009 and May 2019. Data including baseline demographic and clinical characteristics of the patients, preoperative risk factors, radiographic images, and postoperative data were recorded.

Results: Of all patients, six had a failed endograft. The median time to the completion of surgery was 286 (range, 192 to 628 ) min and the median renal ischemia time was 42 (range, 20 to 82) min. Renal artery endarterectomy ( $\mathrm{n}=5)$, reimplantation $(\mathrm{n}=2)$, and an extension graft interposition $(n=2)$ were the additional procedures to ensure kidney perfusion. During a follow-up of 48 months, six patients required dialysis and three were permanent, and the overall mortality rate was $33.3 \%$.

Conclusion: The results of JAA repair become steadily worsened due to the changes in the rate and severity of constitutional risk factors during the last decade. The increasing number of failed endovascular procedures increases the need for a suprarenal clamp. Therefore, failed endograft should be considered an etiological factor and a risk factor for the open repair of JAAs currently.
\end{abstract}

Keywords: Abdominal aortic aneurysm, dialysis, renal artery, surgery.

As the endovascular methods have been used extensively for the repair of infrarenal abdominal aortic aneurysms (AAAs), surgical procedures have been limited to only complicated aneurysms. ${ }^{[1,2]}$ One of the complicated aneurysms which limit the use of endovascular procedures is juxtarenal aneurysms (JAAs) with a hostile neck having the risk for renal artery compromise (Figure $1 \mathrm{a}, \mathrm{b}$ ). ${ }^{[3-5]}$ Open repair of a JAA requires a cross-clamp at the suprarenal level, and there may be a risk for postoperative renal dysfunction. Some reports have suggested that the need for dialysis after JAA surgery is about 3.3\%, and newly developed kidney injury is associated with a higher mortality. ${ }^{[6]} \mathrm{It}$ is, therefore, important to identify and prevent different causes of renal dysfunction.

In the present study, we aimed to evaluate the long-term surgical outcomes of JAAs and to identify perioperative risk factors affecting the results of the open repair of JAAs.

\section{PATIENTS AND METHODS}

In this study, we retrospectively analyzed a total of 21 consecutive patients ( 20 males, 1 female; mean age $64.8 \pm 11.8$ years; range, 31 to 80 years) who underwent open repair of JAAs using a suprarenal clamp at Gaziantep University, Faculty of Medicine, Department of Cardiovascular Surgery between May 2009 and May 2019. A JAA was defined as an AAA which extended to the origin of the renal arteries and required clamping of the aorta above one or both renal arteries due to either the absence of a proximal neck or an endovascular stent/stent-graft endangering infrarenal clamping. Data collection was performed by scanning the electronic surgical records of patients

Received: December 22, 2019 Accepted: January 06, 2020 Published online: March 10, 2020

Correspondence: Doğan Kahraman, MD. Gaziantep Üniversitesi Şahinbey Araştırma ve Uygulama Hastanesi Kalp ve Damar Cerrahisi Kliniği, 27310, Gaziantep, Türkiye. e-mail: drdogankahraman@gmail.com 

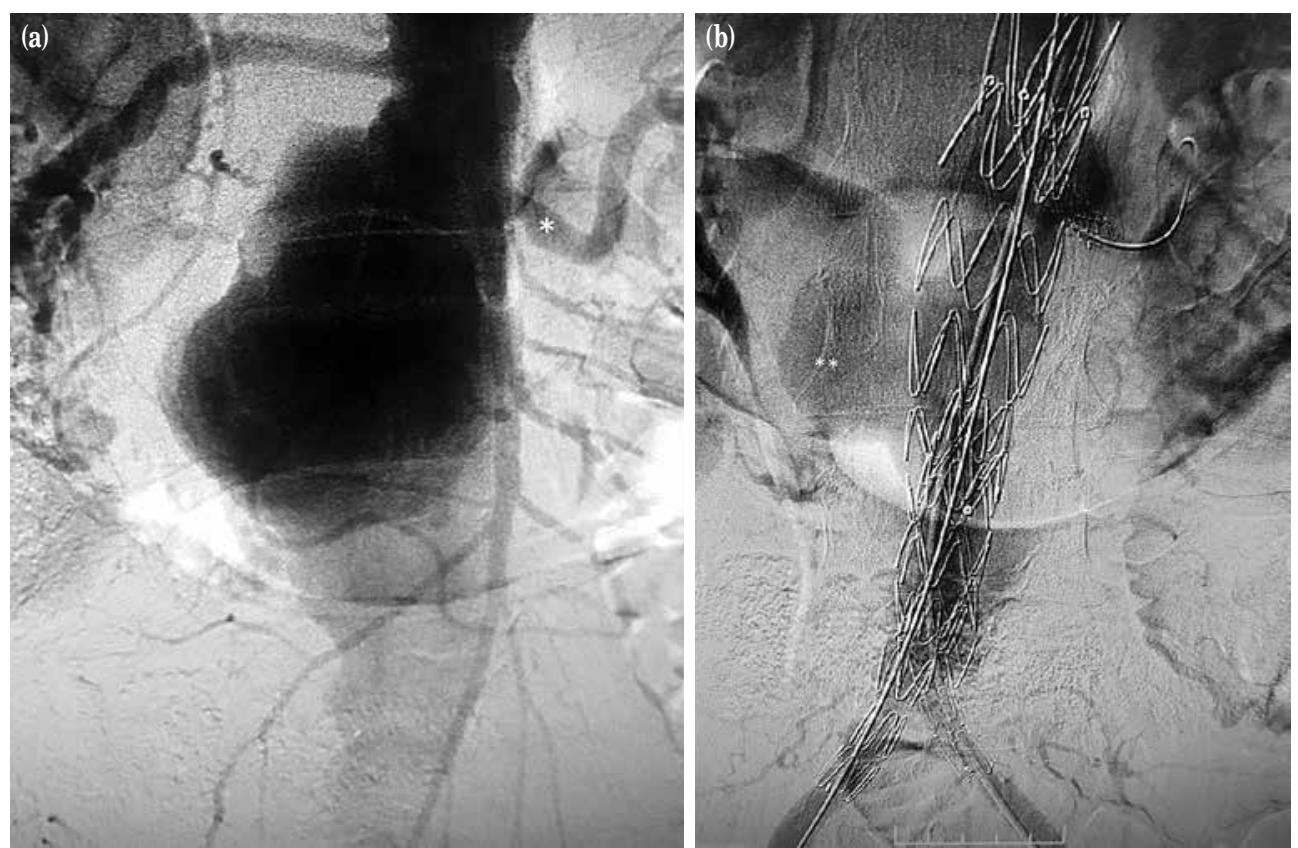

Figure 1. An example of an unsuccessful chimney-endovascular aneurysm repair. A low-rising right renal artery ${ }^{*}$ ) originates from aneurysm wall (a). Final radiographic image (b) shows a type 1 endoleak ${ }^{* *}$ ) even after serial ballooning of proximal endograft. EVAR: endovascular aneurysm repair.

hospitalized for an AAA. The demographic data, perioperative, and follow-up data of the patients who required aortic clamp proximal to the renal artery were recorded for the study. A written informed consent was obtained from each patient. The study protocol was approved by the Gaziantep University Şahinbey Research and Application Hospital Ethics Committee (2019/431). The study was conducted in accordance with the principles of the Declaration of Helsinki.

Preoperative variables, operative measures, and postoperative variables

The patients followed by the nephrology department due to steadily high serum creatinine levels (above $2.5 \mathrm{mg} / \mathrm{dL}$ ) or those on maintenance dialysis were considered as chronic renal failure. Coronary artery disease was defined as having the diagnosis with previous coronary angiography or coronary artery bypass graft surgery. Chronic obstructive pulmonary disease, diabetes, hypertension, previous peripheral vascular procedures (surgical and endovascular), and cerebrovascular disease (transient ischemic attacks, or carotid artery intervention) were extracted from the computerized database with the patients' demographics. Besides, we used the radiographic images to assess the maximum diameter of the aneurysm and anatomic considerations for the suprarenal clamping.
All patients were operated under general anesthesia and through a median laparotomy. The bolus dose of heparin (100 to $150 \mathrm{U} / \mathrm{kg}$ ) was administered after dissecting retroperitoneal tissue and renal arteries. The supra-/interrenal clamp was placed accordingly at a single attempt, followed by distal clamping at the level of iliac arteries. The aneurysm was opened longitudinally, stent graft was removed if present, and bleeding from the origins of lumbar arteries were controlled by sewing with silk sutures. The aorta was transected just distal to the clamp, while leaving a skirt for anastomosis. Freeing of the renal artery origin from the aneurysmal segment, renal artery endarterectomy, removal of renal artery stent, or a graft anastomosis to extend the renal artery to the midline in selected cases were done at this stage, if needed (Figure 2). Each renal artery was perfused immediately with a cold crystalloid solution ( $20 \mathrm{~g}$ mannitol / 1,000 mL lactated Ringer's solution). The proximal end of the graft was anastomosed to the aortic skirt using $3 / 0$ prolene suture as quickly as possible and the proximal clamp was, then, transferred onto the graft. The anastomosis of the free end of the renal artery or the extension graft was performed with a side-clamp. Cold crystalloid perfusion for renoprotection was used in every $15 \mathrm{~min}$ throughout the suprarenal clamping. We finished arterial reconstruction by performing anastomosis the distal part(s) of the graft to the distal aorta, iliac or 


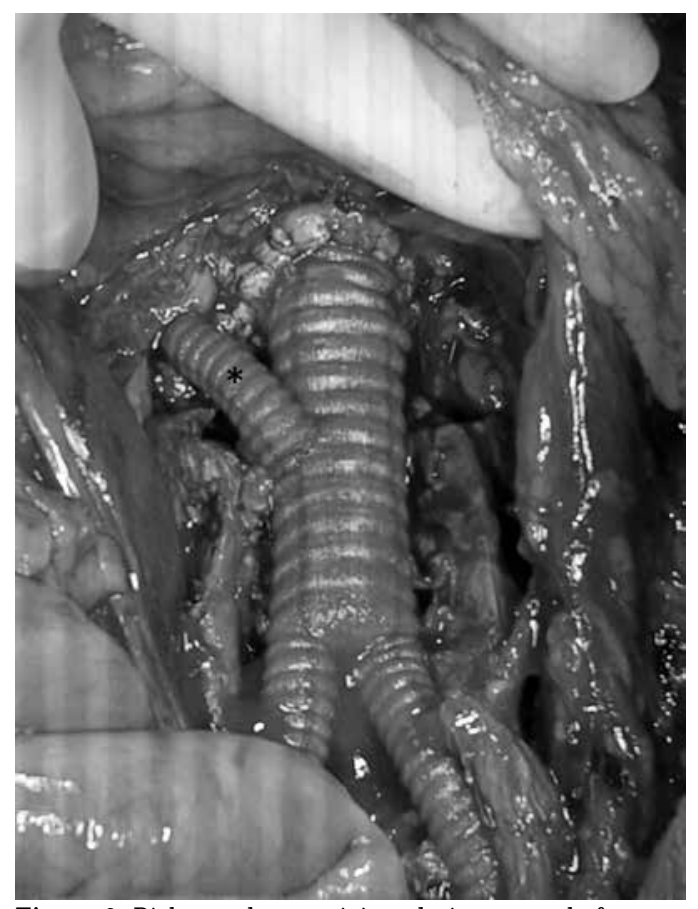

Figure 2. Right renal artery injury during removal of a stent was extended onto aortic graft using an 8-mm Dacron graft $(*)$.

common femoral arteries. Inferior mesenteric artery anastomosis to new aorta was performed, only if sufficient retrograde bleeding was present, and was ligated, otherwise.

Intraoperative variables included renal ischemia time, duration of operation, additional renal artery procedures (i.e., reimplantation, extension graft, endarterectomy), type of aortic reconstruction (i.e., tubular graft, aorto-biiliac or aorto-bifemoral bypass), and level of aortic clamping (suprarenal or interrenal).

The primary outcome measure was mortality, until the last control visit, and the secondary measure was the need for dialysis. Postoperative evaluations included extubation time, and the length of intensive care unit (ICU) and hospital stay.

\section{Statistical analysis}

Statistical analysis was performed using the IBM SPSS version 20.0 software (IBM Corp., Armonk, NY, USA). Continuous variables were expressed in mean \pm standard deviation (SD) and median (min-max), while categorical variables were expressed in number and frequency. The continuous variables with a normal distribution were assessed using the Shapiro-Wilk test. The mean survival time after index surgery was estimated using the KaplanMeier survival analysis ( $95 \%$ confidence interval $[\mathrm{CI}])$. A $p$ value of $<0.05$ was considered statistically significant.

\section{RESULTS}

In all patients, the mean size of JAAs treated in our department over 10 years was $6.3 \pm 1.1 \mathrm{~cm}$. Failed or unsuccessful endovascular AAA repair
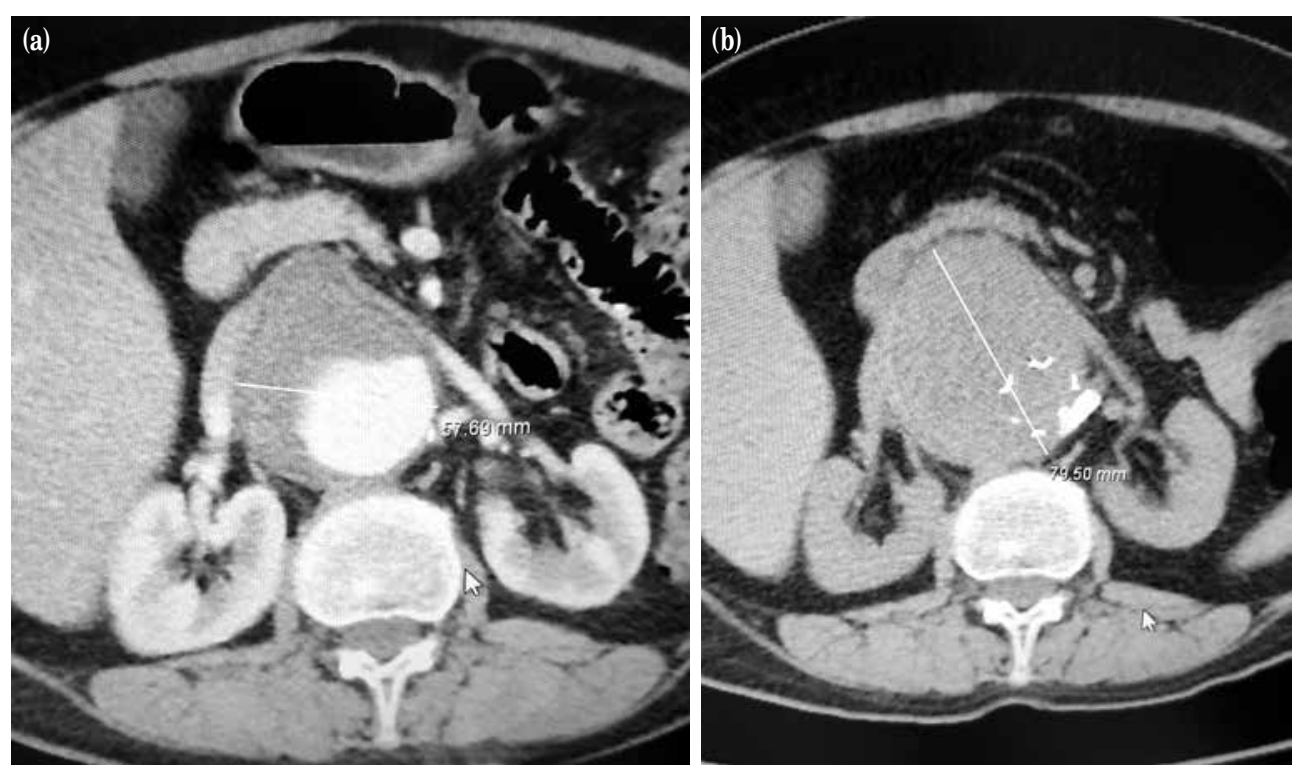

Figure 3. The maximum diameter of an aortic aneurysm measured at same the level (middle of both kidneys) before (a) and six months after EVAR (b) in a patient with chronic renal failure. EVAR: Endovascular aneurysm repair. 


\begin{tabular}{lccccc}
\multicolumn{6}{l}{ Table 1. Baseline demographic and clinical characteristics of patients } \\
\multicolumn{1}{l}{$\mathrm{n}$} & $\%$ & Mean $\pm \mathrm{SD}$ & Median & Min-Max \\
\hline Age (year) & & & $64.8 \pm 11.8$ & & \\
Gender & 20 & 95.2 & & & \\
$\quad$ Male & 1 & 4.8 & & & \\
$\quad$ Female & & & $25.8 \pm 2.1$ & \\
Body mass index $\left(\mathrm{kg} / \mathrm{m}^{2}\right)$ & 9 & 42.9 & & \\
Diabetes & 16 & 76.2 & & \\
Hypertension & 8 & 38.1 & & \\
Hyperlipidemia & 12 & 57.1 & & \\
Chronic obstructive pulmonary disease & 11 & 62.4 & & \\
Coronary artery disease & 1 & 4.8 & & \\
Coronary stent & 3 & 14.3 & & \\
Coronary bypass surgery & 3 & 14.3 & & \\
Ruptured juxtarenal abdominal aortic aneurysm & 3 & 14.3 & & \\
Renal insufficiency & & & & \\
Previous vascular procedures & 6 & 28.6 & & \\
$\quad$ Endovascular abdominal aortic repair & 1 & 4.8 & & \\
$\quad$ Thoracic endovascular aortic repair & 1 & 4.8 & & \\
$\quad$ Femoral-femoral bypass & 2 & 9.5 & & \\
$\quad$ Femoral-popliteal bypass & 2 & 9.5 & & \\
$\quad$ Carotid stent & & & & \\
Serum creatinine (mg/dL) & & & & \\
\hline
\end{tabular}

SD: Standard deviation; Min: Minimum; Max: Maximum.

(EVAR; Figures 1a, b and 3a, b) with ongoing symptoms was present in six patients (28.6\%). Baseline demographics and comorbidities of the patients are shown in Table 1.

Table 2 shows the operative variables. All patients were operated through a median laparotomy and the median time to the completion of surgery was 286 (range, 192 to 628) min and the median renal ischemia time was 42 (range, 20 to 82 ) min. A tube graft was used in three patients (14.3\%), while a bifurcated graft was implanted in 18 patients (85.7\%): an aortobiiliac graft and aorto-bifemoral graft in 16 (76.2\%) and $2(9.5 \%)$ patients, respectively. Seventeen patients
(81\%) were subjected to simple clamping at the suprarenal aorta; however, in four patients (19\%), the aortic clamp was between the renal arteries originating from different levels (Table 3). Additional procedures to ensure postoperative renal perfusion were renal artery re-implantation to the new aorta $(\mathrm{n}=2,9.5 \%)$, interposing an extension graft $(\mathrm{n}=2,9.5 \%$, Figure 2), and endarterectomy $(\mathrm{n}=5,23.8 \%)$.

Table 4 presents the follow-up data. The median postoperative ventilation time was 12 (range, 5 to 31) hours and three patients (14.3\%) were lost during the hospital stay. The overall mortality rate was 33.3\% $(n=7)$ during a mean follow-up of $47.9 \pm 33.6$ months.

\begin{tabular}{lcccc} 
Table 2. 0perative data of patients requiring a suprarenal clamp & & \\
\hline & $\mathrm{n}$ & $\%$ & Median & Min-Max \\
\hline Operation time (min) & & & 286 & $196-628$ \\
Renal ischemia time (min) & & & 42 & $20-82$ \\
Simple suprarenal clamp & 17 & 81 & & \\
Interrenal clamp & 4 & 19 & & \\
Additional renal artery procedure & & & & \\
$\quad$ Extension graft & 2 & 9.5 & & \\
$\quad$ Reimplantation & 2 & 9.5 & & \\
$\quad$ Endarterectomy & 5 & 23.8 & & \\
Type of aortic procedure & & & & \\
$\quad$ Tubular graft & 3 & 14.3 & & \\
$\quad$ Aorto-biiliac graft & 16 & 16.2 & & \\
$\quad$ Aorto-bifemoral graft & 2 & 9.5 & & \\
\hline
\end{tabular}

Min: Minimum; Max: Maximum. 
According to the Kaplan-Meier analysis, the mean survival was $75.6 \pm 10$ months (95\% CI: 56.1-95.2; Figure 4). Hemodialysis was required in six patients including two on maintenance dialysis preoperatively.

\begin{tabular}{lcc} 
Table 3. Reasons for suprarenal clamping & & \\
\hline & $\mathrm{n}$ & $\%$ \\
\hline Short neck & 8 & 38.1 \\
Renal artery arising from aneurysm & 7 & 33.3 \\
Endovacular abdominal aortic repair & 6 & 28.6 \\
\hline
\end{tabular}

\begin{tabular}{lccc}
\multicolumn{4}{c}{ Table 4. Follow-up parameters of patients (during 48 months) } \\
\hline & $\mathrm{n}$ & $\%$ & Mean $\pm \mathrm{SD}$ \\
\hline Extubation time (h) & & & $12.9 \pm 5.8$ \\
Intensive care unit stay (day) & & & $2.9 \pm 1.2$ \\
Hospital stay (day) & & & $9.4 \pm 2.9$ \\
Need for dialysis & 3 & 14.3 & \\
$\quad$ Temporary & 3 & 14.3 & \\
$\quad$ Permanent & 3 & 14.3 & \\
Hospital mortality & 7 & 33.3 & \\
Total mortality & & & $2.9 \pm 1.3$ \\
Highest creatinin (mg/dL) & & & $47.9 \pm 33.6$ \\
Follow-up time (month) & & & \\
Complications & 5 & 23.8 & \\
$\quad$ Pulmonary & 2 & 9.5 & \\
Distal embolism & 1 & 4,8 & \\
Mesenteric ischemia & 2 & 9.5 & \\
$\quad$ Amputation & 1 & 4.8 & \\
$\quad$ Graft infection & 3 & 14.3 & \\
$\quad$ Wound infection & &
\end{tabular}

SD: Standard deviation.

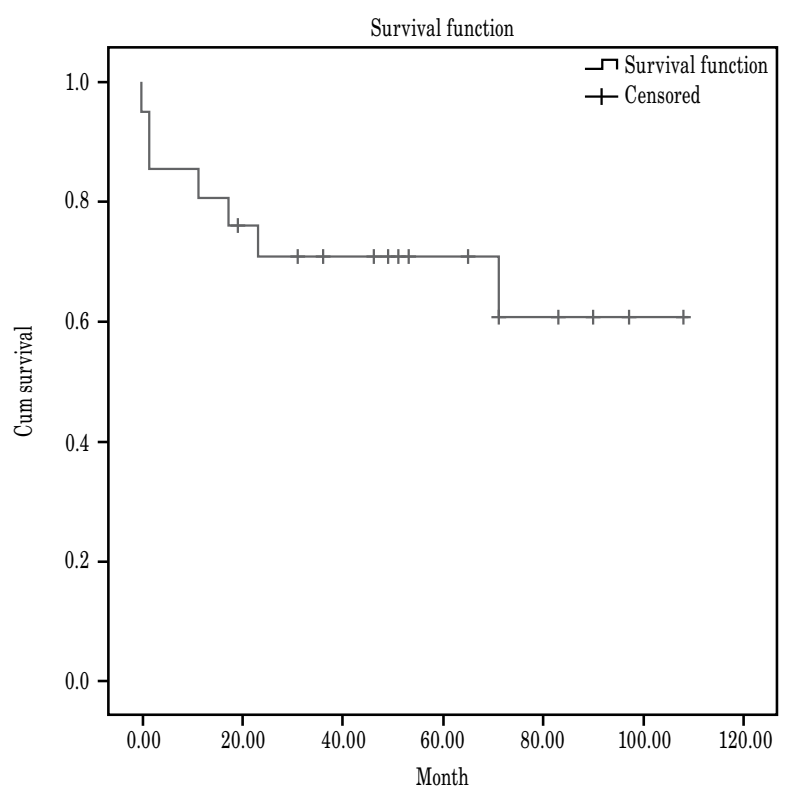

Figure 4. The diagram showing the estimated survival rate of the patient cohort.
Only one new patient continued routine dialysis program after discharge, other two were lost in the ICU and one was followed by the nephrology department after six hemodialysis sessions for the hypervolemic state.

\section{DISCUSSION}

The AAA is seen in 5 to $7 \%$ of individuals over age 60 years and, 2 to $20 \%$ are JAAs or extend to the renal arteries. ${ }^{[7]}$ Surgical repair for symptomatic AAAs has been well-described and effective procedure, although its main disadvantage is the high operating risks, particularly in patients with significant comorbidities. ${ }^{[8-11]}$ Besides, the treatment of JAAs remains more challenging due to inevitable renal ischemia. ${ }^{[10,12]}$ The patients included in our study consisted of elderly patients with comorbidities potentially complicating surgery such as coronary artery disease (33.3\%), chronic obstructive pulmonary disease (57.1\%), and failed EVAR procedures (28.6\%). The hospital mortality was as high as $14.3 \%$; however, it included one early death after ruptured aneurysm repair.

The largest series including 257 patients concluded that open surgery of JAA could be performed with low mortality rates (5.8\%), but with increased renal morbidity (41\%). ${ }^{[13]}$ However, the authors noted a mean

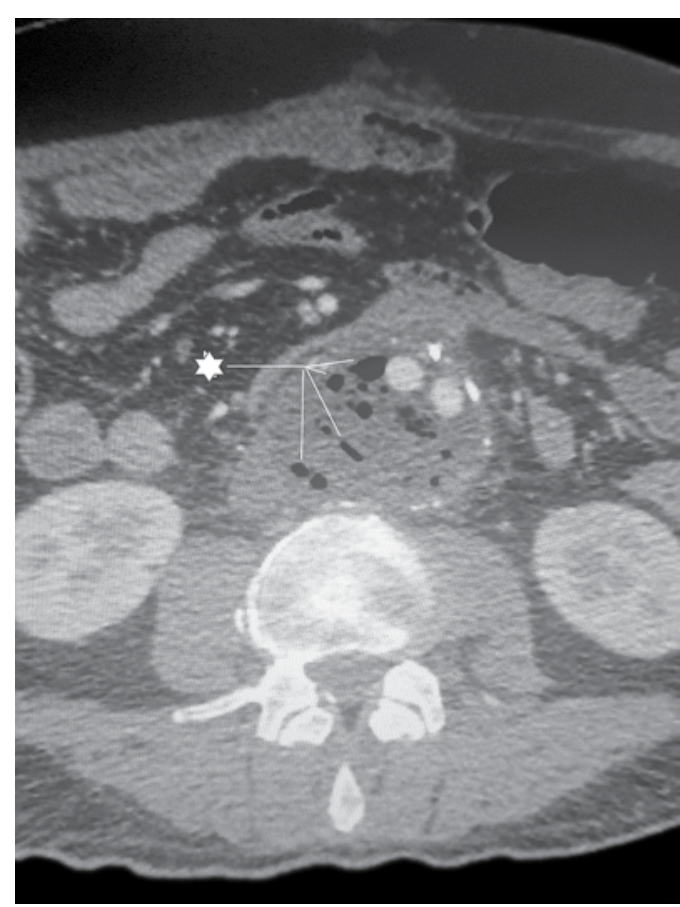

Figure 5. Air bubbles ${ }^{*}$ ) around an infected graft implanted for treatment of juxtarenal aortic aneurysm. 
renal ischemia time of $28 \mathrm{~min}$ during JAA repair, and none of the patients required reconstruction procedures for a troublesome renal artery. A more recent study including 126 patients who required suprarenal cross-clamp was conducted by Knott et al. ${ }^{[7]}$ and they found an operative mortality of $0.8 \%$, renal insufficiency (creatinine increase $>0.5 \mathrm{mg} / \mathrm{dL}$ ) of $18 \%$, and new hemodialysis in $4 \%$ of patients. Notably, they used cold renal instillation (i.e., heparin, mannitol, and lactated Ringer's solution) during suprarenal clamping in 100 patients and the mean duration of renal ischemia was 23 (range, 3 to 90) min that is almost half of our experience. Although the demographics of our patient cohort were almost similar to those in the aforementioned studies, the renal artery ischemia time was remarkably longer. The main differences from these reports are the number of failed EVAR patients requiring suprarenal clamp and the number of additional procedures performed to the renal artery.

Over the last decade, EVAR has become the preferred method of treatment for the infrarenal aortic aneurysms. ${ }^{[14,15]}$ Progressive improvement in endograft technology are associated with improved short-term outcomes, but with a considerable amount of late reinterventions, and widespread use in a higher number of eligible patients. ${ }^{[16,17]}$ The quality of the results is related to individual experience, knowledge about the capacity of special equipment, adequacy and diversity of clinical resources, and appropriate patient selection. Therefore, simple EVAR to infrarenal aneurysms with a hostile neck rather than fenestrated EVAR or chimney/snorkel procedures should be considered to increase the risk of failure (Figure 5). One of the treatment options of failed EVAR is akin to the open repair of JAAs. However, removal of the aortic stent graft and/or concomitant renal stents may require additional interventions to the renal arteries and consequently increase the duration of the renal ischemia. We believe that the high failed EVAR rate in the patients in our study would help to explain the increased mean renal ischemia time, which is reported as $27 \mathrm{~min}$ in the literature, and the higher rate of patients requiring dialysis. ${ }^{[6]}$

Renal perfusion with cold crystalloid perfusate during suprarenal clamping (median: $42 \mathrm{~min}$ ) was applied in all patients, but a median rise of $1.3 \mathrm{mg} / \mathrm{dL}$ serum creatinine level and $28.6 \%$ dialysis requirement were yielded. An ideal level of aortic cross-clamp placement (suprarenal or interrenal) and strategy of renal perfusion remains controversial with conflicting reports in the literature. ${ }^{[7,18,19]}$ Some authors have suggested that postoperative renal dysfunction is not related to renal ischemia time, preoperative renal function, or renal revascularization but cold saline perfusion provides considerable benefits with $0 \%$ postoperative azotemia. ${ }^{[18]}$ In addition to superior results of cold crystalloid perfusate considering renal morbidity, perfusion with isothermic/cold blood seems to be effective to supply oxygen and buffers, prevent cell membrane damage, and reduce cell swelling. ${ }^{[20-22]}$ Others have also reported that the decrease of the estimated glomerular filtration rate is significantly lower in the interrenal clamp than in the suprarenal clamp. ${ }^{[23]}$ Nonetheless, several authors have advocated a simple clamp-and-sew technique which provides approximately $25 \mathrm{~min}$ shorter renal ischemia time than renal perfusion approaches. ${ }^{[7,24]}$ The common opinion in the literature is that renal ischemia exceeding $45 \mathrm{~min}$, with or without renal perfusion strategies, is associated with an increased risk of renal damage. ${ }^{[13,14,25]}$

In our study, renal morbidity, hospital mortality, and mid-term (48 months) mortality seem to be higher compared to previous reports. The reasons that we can infer from this study are the high rate of preoperative comorbidities, the failed EVAR procedure complicating surgery, the concomitant vascular pathologies reflecting diffuse atherosclerosis, and the high rupture rate at presentation. Although many have indicated significant potential advantages of endovascular procedures as a reason to avoid open surgery to treat complex AAAs, the true benchmark for open surgery still remains unclear. ${ }^{[26-28]}$ However, dialysis requirement and mortality for open reconstruction seemed similar to those reported for complex endovascular repairs. ${ }^{[29,30]}$ Indeed, we believe that unsuccessful endovascular procedures tend to increase the complexity of open surgery and, hence, the negative consequences, due to the disproportionate increase in the frequency of use and experience. Also, complications such as pulmonary infections, mesenteric ischemia, limb amputation, peripheral embolism, graft infection are more commonly seen in our patient cohort than those with infrarenal aneurysms.

The present study has several limitations. This single-center study is limited by the relatively small number of patients, and the 10-years interval represented. During this long period, improvements in the operative and perioperative care have been made, which are possible to have improved patient outcomes. We believe that no prospective randomized trials would be obtainable with this small volume of patients in a single-center, and studies with a retrospective design could serve valuable information for the near future. Secondly, this study did not show significant effects 
of some potential factors such as renal perfusion, renal ischemia time, and renal revascularization on renal failure. The reason for this may be due to our strategy which necessitates cold perfusion application in all patients that further prolonged renal ischemic time. Therefore, a sufficient statistical power obtained from a larger sample size could be required to define more predictors. Finally, we did not have complete data including the medical management of comorbidities during follow-up and, thus, the relationship between renal failure and medical management still remains to be elucidated.

In conclusion, the results of this study are consistent with previous reports, showing less favorable outcomes after JAA repair considering renal injury. The discrepancy in prognosis rates seems to be worsened by more extensive pathology, and an increasing number of failed EVAR procedures. As the procedural complexity increases by additional renal artery interventions, ethical considerations before endovascular procedures should be revised individually for low-volume centers. The complexity of surgical procedure due to changing etiological factors requires an accurate preoperative assessment of morphology and operative strategy.

\section{Declaration of conflicting interests}

The authors declared no conflicts of interest with respect to the authorship and/or publication of this article.

\section{Funding}

The authors received no financial support for the research and/or authorship of this article.

\section{REFERENCES}

1. Quiñones-Baldrich WJ, Holden A, Mertens R, Thompson MM, Sawchuk AP, Becquemin JP, et al. Prospective, multicenter experience with the Ventana Fenestrated System for juxtarenal and pararenal aortic aneurysm endovascular repair. J Vasc Surg 2013;58:1-9.

2. Scali ST, McNally MM, Feezor RJ, Chang CK, Waterman AL, Berceli SA, et al. Elective endovascular aortic repair conversion for type Ia endoleak is not associated with increased morbidity or mortality compared with primary juxtarenal aneurysm repair. J Vasc Surg 2014;60:286-94.

3. Oderich GS, Greenberg RK, Farber M, Lyden S, Sanchez L, Fairman R, et al. Results of the United States multicenter prospective study evaluating the Zenith fenestrated endovascular graft for treatment of juxtarenal abdominal aortic aneurysms. J Vasc Surg 2014;60:1420-8.

4. Wooster M, Tanious A, Patel S, Moudgill N, Back M, Shames M. Concomitant Parallel Endografting and Fenestrated Experience in a Regional Aortic Center. Ann Vasc Surg 2017;38:54-8.
5. Lee JT, Lee GK, Chandra V, Dalman RL. Comparison of fenestrated endografts and the snorkel/chimney technique. J Vasc Surg 2014;60:849-56.

6. Jongkind V, Yeung KK, Akkersdijk GJ, Heidsieck D, Reitsma JB, Tangelder GJ, et al. Juxtarenal aortic aneurysm repair. J Vasc Surg 2010;52:760-7.

7. Knott AW, Kalra M, Duncan AA, Reed NR, Bower TC, Hoskin TL, et al. Open repair of juxtarenal aortic aneurysms (JAA) remains a safe option in the era of fenestrated endografts. J Vasc Surg 2008;47:695-701.

8. Schneider JR, Gottner RJ, Golan JF. Supraceliac versus infrarenal aortic cross-clamp for repair of non-ruptured infrarenal and juxtarenal abdominal aortic aneurysm. Cardiovasc Surg 1997;5:279-85.

9. Nordon IM, Hinchliffe RJ, Holt PJ, Loftus IM, Thompson MM. Modern treatment of juxtarenal abdominal aortic aneurysms with fenestrated endografting and open repair--a systematic review. Eur J Vasc Endovasc Surg 2009;38:35-41.

10. Yeung KK, Tangelder GJ, Fung WY, Coveliers HM, Hoksbergen AW, Van Leeuwen PA, et al. Open surgical repair of ruptured juxtarenal aortic aneurysms with and without renal cooling: observations regarding morbidity and mortality. J Vasc Surg 2010;51:551-8.

11. Tsai S, Conrad MF, Patel VI, Kwolek CJ, LaMuraglia GM, Brewster DC, et al. Durability of open repair of juxtarenal abdominal aortic aneurysms. J Vasc Surg 2012;56:2-7.

12. Scarcello E, Serra R, Morrone F, Tarsitano S, Triggiani G, de Franciscis S. Aortic banding and endovascular aneurysm repair in a case of juxtarenal aortic aneurysm with unsuitable infrarenal neck. J Vasc Surg 2012;56:208-11.

13. Jean-Claude JM, Reilly LM, Stoney RJ, Messina LM. Pararenal aortic aneurysms: the future of open aortic aneurysm repair. J Vasc Surg 1999;29:902-12.

14. Landry G, Lau I, Liem T, Mitchell E, Moneta G. Open abdominal aortic aneurysm repair in the endovascular era: effect of clamp site on outcomes. Arch Surg 2009;144:811-6.

15. Desole A, Ferrari A, Tosato F, Milite D. Open Repair for Juxtarenal Aortic Aneurysm: Short and Long-term Results. Ann Vasc Surg 2019;54:161-5.

16. Patel R, Sweeting MJ, Powell JT, Greenhalgh RM; EVAR trial investigators. Endovascular versus open repair of abdominal aortic aneurysm in 15-years' follow-up of the UK endovascular aneurysm repair trial 1 (EVAR trial 1): a randomised controlled trial. Lancet 2016;388:2366-74.

17. Setacci C, Sirignano P, Fineschi V, Frati P, Ricci G, Speziale F. A clinical and ethical review on late results and benefits after EVAR. Ann Med Surg 2017;16:1-6.

18. Yeung KK, Jongkind V, Coveliers HM, Tangelder GJ, Wisselink W. Routine continuous cold perfusion of the kidneys during elective juxtarenal aortic aneurysm repair. Eur J Vasc Endovasc Surg 2008;35:446-51.

19. Shortell CK, Johansson M, Green RM, Illig KA. Optimal operative strategies in repair of juxtarenal abdominal aortic aneurysms. Ann Vasc Surg 2003;17:60-5.

20. Yamashita C, Okada M, Ataka K, Yoshida M, Nohara H, Azami T, et al. Surgical results for thoraco-abdominal 
aneurysm by modified DeBakey method using centrifugal biopump and renal cryopreservation. J Cardiovasc Surg 1998;39:399-404.

21. Köksoy C, LeMaire SA, Curling PE, Raskin SA, Schmittling ZC, Conklin LD, et al. Renal perfusion during thoracoabdominal aortic operations: cold crystalloid is superior to normothermic blood. Ann Thorac Surg 2002;73:730-8.

22. Rehring TF, Hollis HW Jr. Supraceliac aortic shunting for maintenance of antegrade renal perfusion during complex aortic reconstruction. J Am Coll Surg 2003;196:657-60.

23. Kasahara H, Shimizu H, Yozu R. Postoperative renal function after juxtarenal aortic aneurysm repair with simple cross-clamping. Ann Vasc Surg 2013;27:291-8.

24. Chiesa R, Marone EM, Brioschi C, Frigerio S, Tshomba Y, Melissano G. Open repair of pararenal aortic aneurysms: operative management, early results, and risk factor analysis. Ann Vasc Surg 2006;20:739-46.

25. Marrocco-Trischitta MM, Melissano G, Kahlberg A, Vezzoli G, Calori G, Chiesa R. The impact of aortic clamping site on glomerular filtration rate after juxtarenal aneurysm repair. Ann Vasc Surg 2009;23:770-7.

26. Beck AW, Bos WT, Vourliotakis G, Zeebregts CJ, Tielliu IF, Verhoeven EL. Fenestrated and branched endograft repair of juxtarenal aneurysms after previous open aortic reconstruction. J Vasc Surg 2009;49:1387-94.

27. Chinsakchai K, Prapassaro T, Salisatkorn W, Hongku K, Moll FL, Ruangsetakit C, et al. Outcomes of Open Repair, Fenestrated Stent Grafting, and Chimney Grafting in Juxtarenal Abdominal Aortic Aneurysm: Is It Time for a Randomized Trial? Ann Vasc Surg 2019;56:114-23.

28. Chuter TA. Fenestrated and branched stent-grafts for thoracoabdominal, pararenal and juxtarenal aortic aneurysm repair. Semin Vasc Surg 2007;20:90-6.

29. Patel VI, Lancaster RT, Conrad MF, Lamuraglia GM, Kwolek CJ, Brewster DC, et al. Comparable mortality with open repair of complex and infrarenal aortic aneurysm. J Vasc Surg 2011;54:952-9.

30. Kabbani LS, West CA, Viau D, Nypaver TJ, Weaver MR, Barth C, et al. Survival after repair of pararenal and paravisceral abdominal aortic aneurysms. J Vasc Surg 2014;59:1488-94. 